\title{
EDITORIAL
}

\section{Multilevel developmental perspectives toward understanding internalizing psychopathology: Current research and future directions}

\author{
DANTE CICCHETTI $^{a, b}$ AND MISAKI N. NATSUAKI ${ }^{c}$ \\ ${ }^{a}$ University of Minnesota Institute of Child Development; ${ }^{b}$ University of Rochester Mt. Hope Family Center; and ${ }^{c}$ University of \\ California, Riverside
}

This Special Issue of Development and Psychopathology is devoted to a multilevel developmental understanding of internalizing psychopathology. In an earlier Special Issue (Burnette \& Cicchetti, 2012), contributors focused upon a multilevel developmental perspective of externalizing pathology. Internalizing and externalizing expressions of dysfunction comprise one of the most widely agreed upon classifications of behavior disorders in psychopathology research. Over the course of the past three decades, in part due to the scope and magnitude of these problems, developmental psychopathologists have devoted increased research attention to these two broad expressions of dysfunction.

Until relatively recently, the amount of research on internalizing problems has lagged behind that of investigations on externalizing disorders. The contributions to this Special Issue are testimony to some of the exciting multidomain and multilevel advances that have occurred in the developmental approach to internalizing pathology.

Depression, and by extension, internalizing psychopathology more broadly, is a complex, multilevel phenomenon (Cicchetti \& Toth, 1995, 1998; Goodman \& Gottlib, 1999). The developmental psychopathology perspective challenges researchers investigating internalizing problems in children and adults to move beyond identifying isolated cognitive, social-cognitive, affective, interpersonal, and biological aberrations in internalizing pathology, to understanding the processes by which these components have evolved and are integrated within and across the biological and psychological systems of the depressed/anxious person embedded within a multilevel and dynamic cultural and social ecology (Causa-

Address correspondence and reprint requests to: Dante Cicchetti, Institute of Child Development, University of Minnesota, 51 East River Road, Minneapolis, MN 55455; E-mail: cicchett@umn.edu; or Misaki N. Natsuaki, Department of Psychology, University of California, Riverside, 900 University Avenue, Riverside, CA 92521; E-mail: misaki.natsuaki@ucr.edu. dias, 2013; Ciccchetti \& Toth, 1998). In order to comprehend the nature, causes, course, and mechanisms of internalizing psychopathology, an integrative perspective that investigates multiple factors at different levels of dynamic developmental systems is necessary. To fully comprehend the complexity of depression and internalizing psychopathology, it is essential that a multiple levels of analysis approach and an interdisciplinary perspective be incorporated into the research methods of developmental psychopathologists.

Depression and anxiety have been a major topic of inquiry among individuals from a number of scientific disciplines, including not only psychiatrists and clinical and developmental psychologists but also anthropologists, sociologists, geneticists, and neuroscientists. A recent search we conducted via PsychINFO identified over 340,000 articles with a keyword "depression," over 280,000 with "anxiety," and over 16,000 with "internalizing." Clearly, depression and internalizing pathology are already topics that have received abundant attention. Why then should a Special Issue be devoted to them now?

We think that there are several gaps in our knowledge base about internalizing psychopathology that we are striving to fill with this Special Issue. One major gap is that there are relatively few investigations that have charted the processes that unfold over the course of the development of depression and internalizing pathology. There is a pressing need for developmental studies that are concerned with elucidating how, when, and for whom internalizing psychopathology arises, changes, is maintained, recovers, and is prevented in the course of development. Another equally important gap that exists is that there is a relative paucity of interdisciplinary developmental research on depression and internalizing psychopathology. The limited amount of communication between disciplines hampers the ability to undertake such multilevel research.

Because of the growing interest in adopting interdisciplinary research perspectives (Gunnar \& Cicchetti, 2009; Pellmar 
\& Eisenberg, 2000), this is an opportune time to devote a Special Issue of Development and Psychopathology to multilevel developmental perspectives on depression and internalizing psychopathology. It is essential that more emphasis be directed toward the process of development. Development is always the result of interdependence, co-actions, or codetermination among multiple levels of influence (Gottlieb, 2001; Sroufe, 2013). It is not only genes and environments

\section{References}

Burnette, M. L., \& Cicchetti, D. (Eds.). (2012). Multilevel approaches toward understanding the development of antisociality: Current research and future directions [Special Issue]. Development and Psychopathology, 24, 703-1155.

Causadias, J. M. (2013). A roadmap for the integration of culture into developmental psychopathology. Development and Psychopathology, 25, 13751398.

Cicchetti, D., \& Toth, S. L. (1995). Developmental psychopathology and disorders of affect. In D. Cicchetti \& D. J. Cohen (Eds.), Developmental psychopathology: Risk, disorder, and adaptation (Vol. 2, pp. 369-420). New York: Wiley.

Cicchetti, D., \& Toth, S. L. (1998). The development of depression in children and adolescents. American Psychologist, 53, 221-241.

Goodman, S. H., \& Gotlib, I. H. (1999). Risk for psychopathology in the children of depressed mothers: A developmental model for understanding mechanisms of transmission. Psychological Review, 106, 458. but also the cumulative developmental history of the individual that influence how future development will unfold (Sroufe, 2013). The mutually enriching interchanges that have occurred within and across disciplines must continue and be elaborated. The contributions in this Special Issue can serve as a momentum for energizing the conduct of the multilevel, interdisciplinary developmental research on depression and internalizing psychopathology that lies ahead.

Gottlieb, G. (2001). Individual development and evolution: The genesis of novel behavior. New York: Oxford University Press.

Gunnar, M. R., \& Cicchetti, D. (2009). Meeting the challenge of translational research in child psychology. In D. Cicchetti \& M. R. Gunnar (Eds.), Minnesota symposia on child psychology: Vol. 35. Meeting the challenge of translational research in child psychology (pp. 1-27). Hoboken, NJ: Wiley.

Pellmar, T. C., \& Eisenberg, L. (Eds.). (2000). Bridging disciplines in the brain, behavioral, and clinical sciences. Washington, DC: National Academies Press.

Sroufe, L. A. (2013). The promise of developmental psychopathology: Past and present. Development and Psychopathology, 25, 1215-1224. 